\section{HEART FAILURE \\ Epidemiology, aetiology, and prognosis of heart failure}

John J McMurray, Simon Stewart

Clinical Research Initiative in Heart Failure, Wolfson Building,

University of Glasgow, Glasgow, UK
- Comprehensive clinical registries collected in conjunction with clinical trials. These include a large proportion of individuals who were identified on the basis of having both impaired left ventricular systolic dysfunction and signs and symptoms of heart failure.

Within the context of the specific limitations of the type of data available from these studies, the current understanding of the aetiology, epidemiology, and prognostic implications of chronic heart failure are discussed here.

$\mathrm{H}$ eart failure is now recognised as a major and escalating public health problem in industrialised countries with ageing populations. Any attempt to describe the epidemiology, aetiology, and prognosis of heart failure, however, must take account of the difficulty in defining exactly what heart failure is. Though the focus of this article is the symptomatic syndrome it must be remembered that as many patients again may have asymptomatic disease that might be legitimately labelled "heart failure"-for example, asymptomatic left ventricular systolic dysfunction. More comprehensive reviews of the epidemiology and associated burden of heart failure have been published by McMurray and colleagues $^{1}$ and more recently by Cowie and colleagues. $^{2}$

Data relating to the aetiology, epidemiology and prognostic implications of heart failure are principally available from five types of studies:

- Cross sectional and longitudinal follow up surveys of well defined populations. These have almost exclusively focused on those individuals with clinical signs and symptoms indicative of chronic heart failure.

- Cross sectional surveys of individuals who have been medically treated for signs and symptoms of heart failure within a well defined region.

- Echocardiographic surveys of individuals within a well defined population to determine the presence of left ventricular systolic dysfunction.

- Nation wide studies of annual trends in heart failure related hospitalisation identified on the basis of diagnostic coding at discharge.

\section{Epidemiology of heart failure}

\section{Prevalence}

Table 1 summarises the reported prevalence of heart failure according to whether this was estimated from a survey of individuals requiring medical treatment from a general practitioner or from population screening. Despite the wide variation in the reported prevalence of heart failure (undoubtedly caused by differing research methods, in addition to inherent differences in the sociodemographic and risk factor profiles of study cohorts), overall these data demonstrate that the prevalence of clinically overt heart failure increases considerably with age. These data also suggest that the prevalence of heart failure has increased over the past few decades.

Studies of patients visiting a general practitioner There have been several large studies examining the number of patients being treated for signs and symptoms of chronic heart failure by a general practitioner, undertaken in the UK over the past 40 years. Only some of the more recent of these can be reviewed here. For example, Paramshwar and colleagues ${ }^{\mathrm{w} 1}$ examined the clinical records of diuretic treated patients in three general practices in northwest London in 1992 to identify possible cases of heart failure. From a total of 30204 patients, a clinical diagnosis of heart failure was made in 117 cases (46 male and 71 female), giving an overall prevalence of 3.9 cases/1000. Prevalence of heart failure increased considerably with age - in those aged under 65 years the prevalence rate was 0.6 cases $/ 1000$ compared

Table 1 Reported prevalence of heart failure ${ }^{12}$

\begin{tabular}{llll}
\hline Study & Location & Overall prevalence rate & $\begin{array}{l}\text { Prevalence rate in older age } \\
\text { group }\end{array}$ \\
\hline Surveys of treated patients & & & - \\
RCGP (1958) & UK national data & $3 / 1000$ & $65 / 1000(>65$ years $)$ \\
Gibson et al (1966) & Rural cohort, USA & $9-10 / 1000$ & - \\
RCGP (1986) & UK national data & $11 / 1000$ & $28 / 1000(>65$ years) \\
Parameshwar et al (1992) & London, UK & $4 / 1000$ & - \\
Rodeheffer (1993) & Rochester, UK & $3 / 1000(<75$ years) & $80 / 1000(>65$ years) \\
Mair et al (1994) & Liverpool, UK & $15 / 1000$ & $74 / 1000(65-74$ years) \\
RCGP (1995) & UK national data & $9 / 1000$ & $40-60 / 1000(>70$ years) \\
Clarke et al (1995) & Nottinghamshire, UK & $8-16 / 1000$ & $30-50 / 1000(>62$ years) \\
Population screening & & & $35 / 1000(65-74$ years) \\
Droller and Pemberton (1953) & Sheffield, UK & - & $23 / 1000(60-79$ years) \\
Garrison et al (1966) & Georgia, USA & $21 / 1000(45-74$ years) & $80-170 / 1000(>67$ years) \\
Framingham (1971) & Framingham, USA & $3 / 1000(<63$ years) & $130 / 1000(>67$ years) \\
Landahl et al (1984) & Sweden (males only) & $3 / 1000(<75$ years) & $80 / 1000(>65$ years) \\
Eriksson et al (1989) & Gothenburg, Sweden & - & $80 / 1000(>65$ years) \\
NHANES (1992) & USA national data & $20 / 1000$ & $74 / 1000(65-74$ years) \\
Cardiovascular health study (1993) & USA national data & $20 / 1000$ & $9 / 1000(25-74$ years) \\
RCGP (1995) & UK national data & & \\
\hline
\end{tabular}

Correspondence to:

Professor John J

McMurray, The

Clinical Research

Initiative in Heart

Failure, Wolfson

Building, University of

Glasgow, Glasgow,

United Kingdom,

G12 8QQ, UK

email:

J.McMurray@bio.gla.ac.uk 
to 28 cases/1000 in those aged over 65 years. However, objective investigation of left ventricular function had been undertaken in less than one third of these patients. Using similar methods, Mair and colleagues ${ }^{\mathrm{w} 2}$ identified a total of 266 cases of heart failure from 17400 patients within two general practices in Liverpool. Undertaken in 1994, the overall prevalence rate was 15 cases/ 1000 patients with 80 cases $/ 1000$ in those aged $\geqslant 65$ years.

More recently, Clarke and colleagues ${ }^{\mathrm{w} 3}$ reported an even larger survey of heart failure based on similar methods and including analysis of prescription of loop diuretics for all residents of the English county of Nottinghamshire. They estimated that between 13017 and 26214 patients had been prescribed frusemide (furosemide) in this region of central England. Case note review of a random sample of those patients receiving such treatment found that $56 \%$ were being treated for heart failure. On this basis they calculated an overall prevalence rate of 8-16 cases/1000. Once again, heart failure prevalence increased with advancing age with the rate increasing to between 40-60 cases $/ 1000$ among those aged $\geqslant 70$ years.

\section{Population studies based on clinical criteria.}

There are now many population studies of heart failure and only some can be reviewed here. At entry into the Framingham study, 17 of 5209 persons (3 cases/1000) screened for heart failure on the basis of clinical criteria were thought to have heart failure; all were less that 63 years of age. ${ }^{\mathrm{w} 4}$ After 34 years follow up, prevalence rates increased as the cohort aged. The estimated prevalence of heart failure in the age groups $50-59,60-69,70-79$, and $\geqslant 80$ years was $8,23,49$, and 91 cases/1000 persons respectively. ${ }^{3}$ NHANES-1 (national health and nutrition examination survey) reported the heart failure prevalence rate within the US population. Based on self reporting, and a clinical scoring system, this study screened 14407 persons of both sexes, aged 25-47 years, between 1971 and 1975, with detailed evaluation of only 6913 subjects and reported a prevalence rate of 20 cases/1000. ${ }^{\text {w5 }}$ The study of men born in 1913 examined the prevalence of heart failure in a cohort of 855 Swedish men

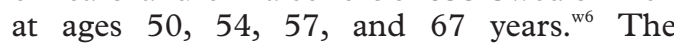
prevalence rate of "manifest" heart failure rose dramatically from 21 cases/1000 at age 50 years to 130 cases/1000 at age 67 years.

Prevalence of left ventricular systolic dysfunction In only a few of the two types of prevalence study described above was objective evidence of cardiac dysfunction obtained. Consequently, it is unclear whether all patients really had heart failure and, if they did, what the cause of heart failure was. There have, however, been four recent estimates of the population prevalence of left ventricular systolic dysfunction as determined by echocardiography emanating from Scotland, ${ }^{4}$ the Netherlands, England, and Finland.

The Scottish study targeted a representative cohort of 2000 persons aged 25-74 years living north of the River Clyde in Glasgow. Of those selected 1640 (83\%) underwent a detailed assessment of their cardiovascular status and underwent echocardiography. Left ventricular systolic dysfunction was defined as a left ventricular ejection fraction $(\mathrm{LVEF}) \leqslant 30 \%$. The overall prevalence of left ventricular systolic dysfunction using this criterion was $2.9 \%$. Concurrent symptoms of heart failure were found in $1.5 \%$ of the cohort, while the remaining $1.4 \%$ were asymptomatic. Prevalence was both greater in men and increased with age: in men aged $65-74$ years it was $6.4 \%$ and in age matched women $4.9 \%$.

The Rotterdam study in the Netherlands, though examining individuals aged 55-74 years, reported similar findings. Overall the prevalence of left ventricular systolic dysfunction, defined in this case as fractional shortening of $\leqslant 25 \%$, was $5.5 \%$ in men and $2.2 \%$ in women. ${ }^{\text {w7 }}$

The Helsinki ageing study describes clinical and echocardiographic findings in 501 subjects (367 female) aged 75-86 years. ${ }^{\text {w8 }}$ The prevalence of heart failure, based on clinical criteria, was $8.2 \%$ overall (41 of 501 ) and $6.8 \%, 10 \%$, and $8.1 \%$ in those aged 75,80 , and 85 years, respectively. These individuals had a high prevalence of moderate or severe mitral or aortic valve disease $(51 \%)$, ischaemic heart disease (54\%), and hypertension (54\%). However, of the 41 subjects with "heart failure" only 11 (28\%) had significant left ventricular systolic dysfunction (diagnosed by the combined presence of fractional shortening $<25 \%$ and left ventricular dilation), and in 20 cases no echocardiographic abnormality was identified. Of the 460 without symptoms of heart failure $43(9 \%)$ also had left ventricular systolic dysfunction. The overall prevalence of left ventricular systolic dysfunction was therefore $10.8 \%$ (95\% confidence interval (CI) $8.2 \%$ to $13.8 \%)$.

More recently, Morgan and colleagues ${ }^{\mathrm{w} 9}$ studied 817 individuals aged $70-84$ years selected from two general practices in Southampton, England. Left ventricular function was assessed qualitatively as normal, mild, moderate or severe systolic dysfunction. The overall prevalence of all grades of dysfunction was $7.5 \%$ (95\% CI 5.8\% to $9.5 \%$ ). Prevalence of left ventricular dysfunction doubled between the ages of 70-74 years and $>80$ years.

Preserved left ventricular systolic function

One of the most controversial issues pertaining to the subject of heart failure at present is the occurrence of the syndrome in patients with preserved left ventricular systolic function (and no other obvious cause, such as valve disease). A full discussion of this topic is beyond the scope of this article. There are, however, two recent studies of this type of heart failure. The Olmsted county study, Minnesota, found that $43 \%$ of patients with chronic heart failure had an LVEF $\geqslant 50 \%$. ${ }^{5}$ Similarly, the Framingham investigators found that $51 \%$ of their cohort with heart failure had an LVEF of $\geqslant 50 \%$ (see also Helsinki ageing study above) ${ }^{6}$ 


\section{Incidence}

There is much less known about the incidence than the prevalence of heart failure. Table 2 shows reported incidence rates from the largest population based studies. The most detailed incidence data emanate from the Framingham heart study. ${ }^{3}$ Like other population based prevalence studies heart failure was defined according to a clinical scoring system. The only "cardiac" investigation was chest radiography. At 34 years follow up, the incidence of heart failure was approximately 2 new cases/1000 in persons aged $45-54$ years, increasing to 40 new cases/1000 in men aged 85-94 years. Using similar criteria, the study of men born in 1913 reported incidence rates of "manifest" heart failure of $1.5,4.3$, and 10.2 new cases/1000 in men aged 50-54, 55-60, and 61-67 years, respectively. ${ }^{\mathrm{w} 6}$ The Rochester epidemiology project also reported the incidence of heart failure in a US population during 1981 in persons aged $0-74$ years. ${ }^{\text {w10 }}$ The annual incidence was 1.1 new cases/1000. Once again incidence was higher in men compared to women (1.57 v 0.71 cases $/ 1000$, respectively). It also increased with age, the rate of new cases increasing from $0.76 / 1000$ in men aged $45-49$ years to $1.6 / 1000$ in men aged $65-69$ years.

The most recent incidence study was reported by Cowie and colleagues from the Hillingdon district of London with a population of approximately $150000 .{ }^{7}$ In a 15 month period, 122 patients were referred to a special heart failure clinic. This represented an annual referral rate of 6.5/1000 population. Using a broad definition of heart failure, only $29 \%$ of these patients were clearly diagnosed as having heart failure (annual incidence 1.85/1000 population).

\section{Heart failure admissions}

A different type of epidemiological information comes from reports of heart failure related hospital admissions on a country to country basis; however, these also need to be interpreted with some caution because of their retrospective nature and variations in coding practices and changing admission thresholds over time. Figure 1 shows the reported hospitalisation rates from Scotland, ${ }^{8}$ Spain, the USA, Sweden, New Zealand, and the Netherlands for the period 1978 to 1993. As such, hospitalisation for heart failure appears to be a growing problem on a global scale. For example, studies undertaken in the UK suggest that in the early $1990 \mathrm{~s} 0.2 \%$ of the population were hospitalised for heart failure per annum and that such admissions accounted for more than $5 \%$ of adult general medicine and geriatric hospital admissions - outnumbering those associated with acute myocardial infarction. ${ }^{8}$ In the USA heart failure continues to be the most common cause of hospitalisation in people over the age of 65 years. ${ }^{9}$

An admission for heart failure is frequently prolonged and in many cases followed by readmission within a short period of time. For example, in the UK the mean length of stay for a heart failure related admission in 1990 was 11.4 days on acute medical wards and 28.5
Table 2 Reported incidence of heart failure. ${ }^{12}$

\begin{tabular}{llll}
\hline Study & Location & $\begin{array}{l}\text { Incidence rate } \\
\text { (whole population) }\end{array}$ & $\begin{array}{l}\text { Incidence rate in older } \\
\text { age groups }\end{array}$ \\
\hline Eriksson et al (1989) & $\begin{array}{l}\text { Sweden (men born in } \\
\text { 1913) }\end{array}$ & - & $10 / 1000$ (61-67 years) \\
Remes et al (1992) & Eastern Finland & $1-4 / 1000(45-74$ years) & $8 / 1000$ (> 65 years) \\
Ho et al $(1993)$ & Framingham, USA & $2 / 1000$ & - \\
Rodeheffer et al (1993) & Rochester, USA & $1 / 1000(<75$ years) & $16 / 1000$ (> 65 years) \\
Cowie et al (1999) & London, UK & $1 / 1000$ & $12 / 1000$ (> 85 years) \\
\hline
\end{tabular}

days on acute geriatric wards. Within the UK about one third of patients are readmitted within 12 months of discharge, while the same proportion are reportedly readmitted within six months in the USA. ${ }^{8} 9$ Such readmission rates are usually higher than the other major causes of hospitalisation, including stroke, hip fracture, and respiratory disease. Moreover, although there is evidence to suggest that an increasing number of heart failure patients are surviving a heart failure related hospital admission, there is a parallel decrease in the number of patients who are discharged on an independent basis to their own homes. On a sex specific basis, men tend to be younger than women when admitted for the first time with heart failure, but because of greater female longevity, the number of male and female admissions are roughly equal.

\section{Cost of heart failure}

In any health care system, hospital admissions represent a disproportionate component of total health care expenditure. Not surprisingly, considering the high rates of hospitalisation for heart failure and the ongoing treatment and care it requires, the overall management of heart failure requires a significant amount of health care expenditure in industrialised nations. Figure 2 shows that heart failure is reported to consume $1-2 \%$ of health care expenditure in a number of industrialised countries. ${ }^{1}$ Moreover, considering the increasing rates of hospitalisation it is likely that these reported estimates fall short of the current burden of heart failure.

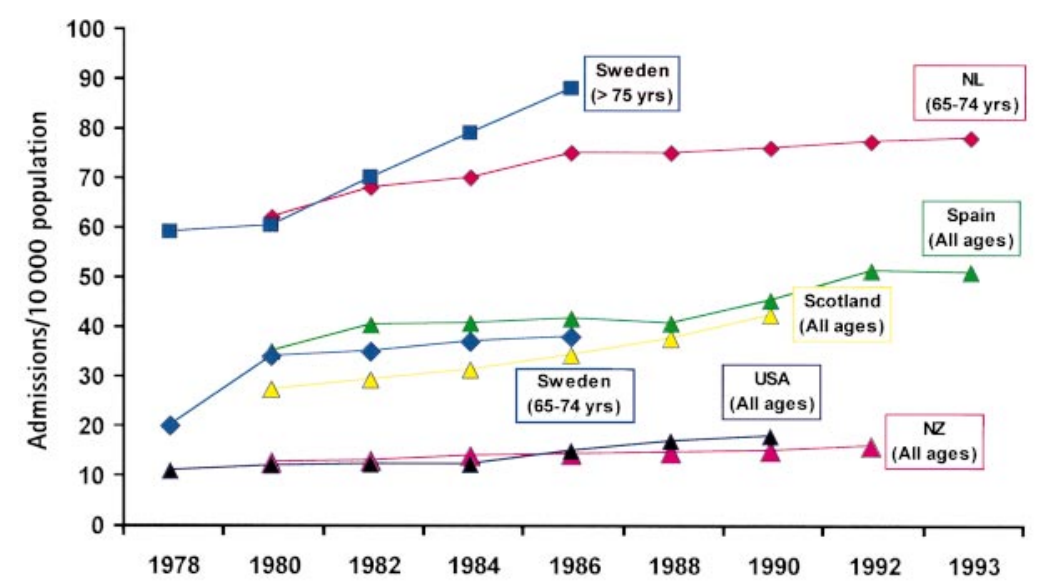

Figure 1: Comparison of heart failure admissions rates per annum (recorded hospital admissions/10 000 population at risk) in western developed countries 1978 to 1993. Adapted from data in McMurray et al. 


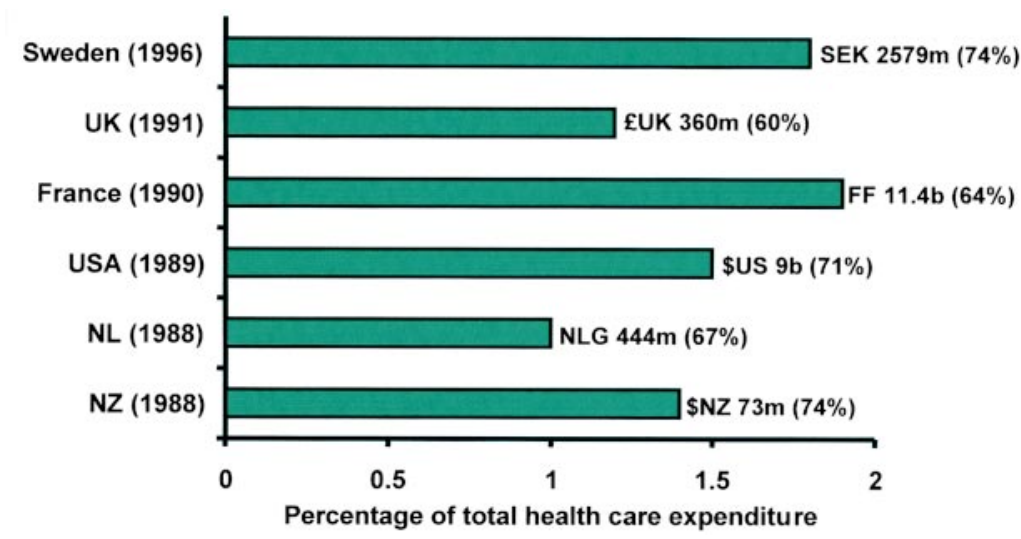

Figure 2: Cost of chronic heart failure compared with the total health expenditure in Sweden, the UK, France, the USA, the Netherlands, and New Zealand. The figures represent the component of hospital costs contributing to total expenditure quoted in the local currency and (in parentheses) as a proportion of total health care expenditure for that country. Adapted from data in McMurray et al.

\section{Aetiology of heart failure}

In western developed countries, coronary artery disease, either alone or in combination with hypertension, seems to be the most common cause of heart failure. It is, however, very difficult to be certain what is the primary aetiology of heart failure in a patient with multiple potential causes (for example, coronary artery disease, hypertension, diabetes mellitus, atrial fibrillation, etc). Furthermore, even the absence of overt hypertension in a patient presenting with heart failure does not rule out an important aetiological role in the past, with normalisation of blood pressure as the patient develops pump failure. Even in those with suspected coronary artery disease the diagnosis is not always correct and in the absence of coronary angiography must remain presumed rather than confirmed. In this context, even coronary angiography has its limitations in identifying atherosclerotic disease.

The initial cohort of the Framingham heart study was monitored until 1965; hypertension appeared to be the most common cause of heart failure, being identified as the primary cause in $30 \%$ of men and $20 \%$ of women and a cofactor in a further $33 \%$ and $25 \%$, respectively. Moreover, electrocardiographic evidence of left ven-

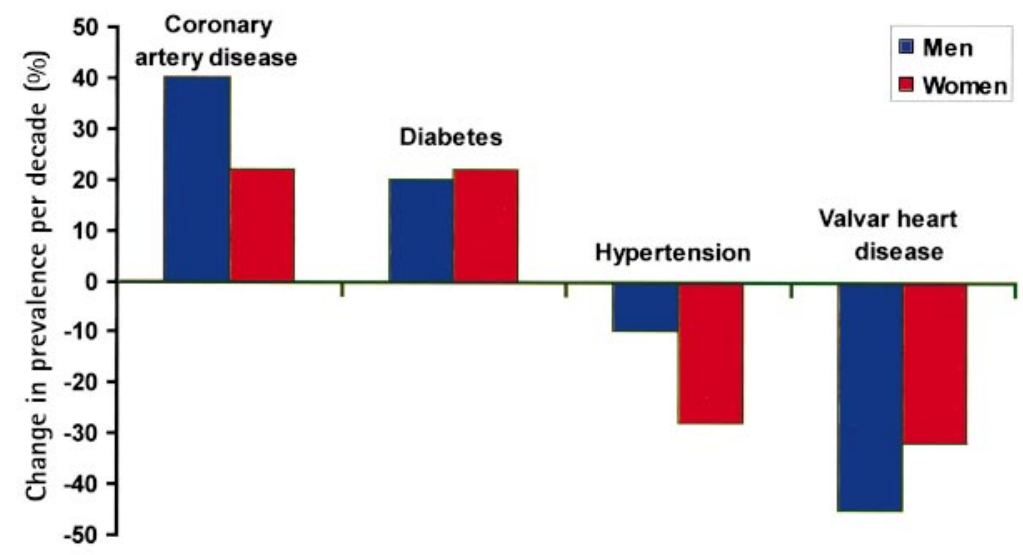

Figure 3: Change in causal factors for heart failure in the Framingham heart study during the period 1950 to 1987. Adapted from data reported by Kannel WB et al. ${ }^{10}$
Common precursors of chronic heart failure

- Coronary artery disease (for example, consequent upon acute myocardial infarction)

- Chronic hypertension

- Cardiomyopathy (for example, dilated, hypertrophic, alcoholic, and idiopathic)

- Valve dysfunction (for example, diseases of the aortic and mitral valve)

- Cardiac arrhythmias/conduction disturbance (for example, heart block and atrial fibrillation)

- Pericardial disease (for example, constrictive pericarditis)

- Infection (for example, rheumatic fever, Chagas disease, viral myocarditis, and HIV)

tricular hypertrophy in the presence of hypertension carried an approximate 15 fold increased risk of developing heart failure. In the subsequent years of follow up, however, coronary heart disease became increasingly prevalent before the development of heart failure and, as the identified cause of new cases of heart failure, increased from $22 \%$ in the 1950 s to almost $70 \%$ in the 1970 s. ${ }^{\mathrm{w} 11}$ During this period, the relative contribution of hypertension and valvar heart disease declined dramatically. Figure 3 is a summary of the changing association of coronary artery disease, hypertension, diabetes, and valvar heart disease with the subsequent development of heart failure over the period 1950 to $1987 .{ }^{10}$ As such there was an approximate $5 \%$ and $30 \%$ decline in the prevalence per decade of hypertension during this period among men and women, respectively. The declining contribution of hypertension most probably reflects the introduction of antihypertensive treatment; the parallel decline in the prevalence of left ventricular hypertrophy supports this supposition. It is also probable that during this same period, progressively greater accuracy in determining the presence of coronary heart disease contributed to its increasing importance in this regard.

As noted above, however, any interpretation of the Framingham data has to consider the fact that heart failure was identified on clinical criteria alone and undoubtedly included individuals without associated left ventricular systolic dysfunction. Conversely, the large scale clinical trials have largely recruited patients who have reduced left ventricular ejection fractions and applied an extensive list of exclusion criteria. Table 3 is a summary of the most commonly attributed causes and associates of heart failure in a number of clinical trials and registries. ${ }^{11-17}$ As such it demonstrates that coronary artery disease appears to be the most common underlying cause of heart failure, consistent with the more recent Framingham experience. 


\begin{tabular}{|c|c|c|c|c|c|c|c|}
\hline & \multicolumn{5}{|c|}{ Clinical trials } & \multicolumn{2}{|l|}{ Registers } \\
\hline & $\begin{array}{l}\text { SOLVD } \\
1991\end{array}$ & $\begin{array}{l}\text { DIG Study } \\
1997\end{array}$ & $\begin{array}{l}\text { MERIT-HF } \\
1999\end{array}$ & $\begin{array}{l}\text { ATLAS } \\
1999\end{array}$ & $\begin{array}{l}\text { RALES } \\
1999\end{array}$ & $\begin{array}{l}\text { SOLVD } \\
1992\end{array}$ & $\begin{array}{l}\text { SPICE } \\
1999\end{array}$ \\
\hline Size of cohort & 2569 & 6800 & 3991 & 3192 & 1663 & 6273 & 9580 \\
\hline Mean age & 61 & 64 & 64 & 64 & 65 & 62 & 66 \\
\hline Male (\%) & 80 & 78 & 78 & 79 & 73 & 74 & 74 \\
\hline \multicolumn{8}{|c|}{ Aetiology of heart failure } \\
\hline Ischaemic & $71 \%$ & $70 \%$ & $66 \%$ & $64 \%$ & $54 \%$ & $69 \%$ & $63 \%$ \\
\hline Non-ischaemic & - & $29 \%$ & $34 \%$ & $35 \%$ & $46 \%$ & $31 \%$ & - \\
\hline Hypertensive & - & $(9 \%)$ & - & $(20 \%)$ & - & $7 \%$ & $4 \%$ \\
\hline IDCM & $18 \%$ & $(15 \%)$ & - & $(28 \%)$ & - & $13 \%$ & $17 \%$ \\
\hline Valvar & - & - & - & $(6 \%)$ & - & - & $5 \%$ \\
\hline Other & - & $6 \%$ & - & - & - & $11 \%$ & - \\
\hline Unknown & - & - & - & & - & - & $6 \%$ \\
\hline \multicolumn{8}{|l|}{ Comorbidity } \\
\hline Hypertension & $42 \%$ & - & $44 \%$ & $46 \%$ & - & $43 \%$ & $27 \%$ \\
\hline Diabetes & $26 \%$ & - & $25 \%$ & $29 \%$ & - & $23 \%$ & - \\
\hline Atrial fibrillation & $10 \%$ & - & $17 \%$ & - & - & $14 \%$ & - \\
\hline Current angina & $37 \%$ & - & - & $27 \%$ & - & & - \\
\hline Respiratory disease & $26 \%$ & - & - & - & - & $15 \%$ & - \\
\hline
\end{tabular}

In the study of left ventricular function in North Glasgow, ${ }^{4} 95 \%$ v $71 \%$ of symptomatic and asymptomatic individuals with definite left ventricular systolic dysfunction had evidence of coronary artery disease $(p=0.04)$. Those individuals with symptomatic heart failure were also more likely to have a past myocardial infarction $(50 \% v 14 \% ; \mathrm{p}=0.01)$ and concurrent angina $(62 \%$ v $43 \%$; $\mathrm{p}=0.02)$. Hypertension (80\%) and valvar heart disease $(25 \%)$ were also more prevalent in those individuals with both clinical and echocardiographically determined heart failure compared to the remainder of the cohort, including those with asymptomatic left ventricular dysfunction $(67 \%$ and $0 \%$, respectively). ${ }^{4}$ One recent study, however, reports an unknown aetiology for heart failure in a disturbingly high proportion of cases. ${ }^{7}$

Therefore, the aetiological importance of many of the associated causes of heart failure will depend on both the age cohorts examined and the type of criteria used to determine the presence of heart failure.

\section{Prognosis}

Heart failure, irrespective of whether it has been detected on the basis of being actively treated (for example, during a hospital admission) or in otherwise asymptomatic individuals, is a lethal condition.

There are some data to suggest that heart failure related mortality is comparable to that of cancer. For example, in the original and subsequent Framingham cohort, the probability of someone with a diagnosis of heart failure dying within five years was $62 \%$ and $75 \%$ in men and $38 \%$ and $42 \%$ in women, respectively. ${ }^{18}$ In comparison, five year survival for all cancers among men and women in the US during the same period was approximately $50 \%$. The general applicability of these data is, however, limited by the few events recorded overall, the relative homogeneity of the Framingham population, and the exclusion of older individuals. The Rochester epidemiology project has also described the prognosis in 107 patients presenting to associated hospitals with new onset heart failure in 1981, and 141 patients presenting in 1991. The median follow up in these cohorts was 1061 and 1233 days, respectively. The mean age of the 1981 patients was 75 years rising to 77 years in 1991. The one year and five year mortality was, respectively, $28 \%$ and $66 \%$ in the 1981 cohort and $23 \%$ and $67 \%$ in the 1991 cohort. $^{\mathrm{w} 12}$ In other words, though the same diagnostic criteria used in the Framingham study were used in the Rochester project, the prognosis was somewhat better in the latter.

The only other large, representative, epidemiological study reporting long term outcome in patients with heart failure is the NHANESI. ${ }^{\mathrm{w5}}$ The initial programme evaluated 14407 adults aged 25 and 74 years in the USA between 1971 and 1975. Follow up studies were carried out in 1982-84 and again in 1986 (for those aged $>55$ years and alive during the 1982-84 review). The estimated 10 year mortality in subjects aged $25-74$ years with self reported heart failure was $42.8 \%(49.8 \%$ in men and $36 \%$ in women). Mortality in those aged $65-74$ years was $65.4 \%(71.8 \%$ and $59.5 \%$ in men and women, respectively). These mortality rates are considerably lower than those observed in Framingham. The patients in NHANES-I were noninstitutionalised and their heart failure was self reported. Follow up was incomplete. NHANES-I was also carried out in a more recent time period than Framingham when prognosis in heart failure patients may have improved. Framingham investigators in 1993 looked at patients developing heart failure in the period 1948 to 1988 and the Rochester investigators in the period 1981 to 1991 . In both of these studies no temporal change in prognosis was identified.

All three of these studies describe a mixed population of patients, some of whom had systolic left ventricular dysfunction and others who did not. The true contribution of heart failure to overall mortality or coronary artery disease related mortality is almost 
certainly underestimated. Although heart failure is highly prevalent among the elderly, representing the terminal manifestation of a number of cardiovascular disease states, and has been shown to be associated with extremely poor survival rates, official statistics continue to attribute only a small proportion of deaths to this syndrome. This reflects a common policy of coding the cause of death as the underlying aetiology (for example, coronary artery disease rather than heart failure itself).

\section{Future burden of heart failure}

Despite a decline in age adjusted mortality from coronary heart disease (CHD) in developed countries overall, the number of patients with chronic CHD is increasing. This is principally the result of two separate trends. Firstly, the proportion of elderly in the population is increasing rapidly and these subjects have the highest incidence of $\mathrm{CHD}$ and hypertension. Secondly, survival in those with coronary artery disease is improving. In particular, it has been shown that survival after acute myocardial infarction has increased notably over the past decade, at least in part because of better medical treatment. ${ }^{19}$ As coronary artery disease is the most powerful risk factor for heart failure (and its most important precursor) it is likely that the aforementioned trends will lead to an increase its future prevalence. Chronic heart failure may, therefore, become a more common manifestation of chronic heart disease and contribute to many more deaths. Two formal projections of the future burden of heart failure have been undertaken in respect to the Netherlands ${ }^{20}$ and Australia. ${ }^{\text {w13 }}$ For example, an analysis of demographic trends in the Netherlands has predicted that the prevalence of heart failure caused by coronary heart disease will rise by approximately $70 \%$ from 1985 to 2010 .

1. McMurray JJ, Petrie MC, Murdoch DR, et al. Clinical epidemiology of heart failure: public and private health burden. Eur Heart J 1998;19:P9-16.

2. Cowie MR. Annotated references in epidemiology. Eur J Heart Failure 1999;1:101-7.

- An update of a comprehensive overview of the epidemiology of heart failure published by the same author in 1997.

3. Ho KK, Pinsky JL, Kannel WB, et al. The epidemiology of heart failure: The Framingham study. J Am Coll Cardiol

1993;22:6A-13A.

- A follow up report of the classic incidence and natural history study conducted in a community in northeastern USA, starting in 1946 and continuing to this day. It describes the long term follow up of a sizeable cohort of individuals with chronic heart failure.

4. McDonagh TA, Morrison CE, Lawrence A, et al Symptomatic and asymptomatic left-ventricular systolic dysfunction in an urban population. Lancet

1997;350:829-33.

- The first population survey of men and women to report the prevalence estimates of left ventricular systolic dysfunction using echocardiography. It was carried out in north Glasgow, which has a very high prevalence of coronary artery disease and hypertension.

5. Senni M, Tribouilloy CM, Rodeheffer RJ. Congestive heart failure in the community: a study of all incident cases in Olmsted County, Minnesota, in 1991. Circulation 1998;98:2282-9.

6. Vasan RS, Larson MG, Benjamin EJ, et al. Congestive heart failure in subjects with normal versus reduced left

\section{Summary}

- Heart failure represents a major and escalating public health problem

- The overall prevalence of clinically identified heart failure is estimated to be 3-20 cases/ 1000 population, but rises to $>100$ cases/ 1000 population in those aged $\geqslant 65$ years.

- Prevalence of confirmed left ventricular systolic dysfunction also increases with age and is more common in men. However, these rates are far less than reported for the syndrome of heart failure.

- The overall annual incidence of clinically overt heart failure in middle aged men and women is approximately $0.1-0.2 \%$.

However, with each additional decade of life there is an approximate doubling of this rate and the incidence of heart failure in those aged $>85$ years is approximately $2-3 \%$.

- Although reported incidence rates are higher in men than women, greater longevity in women tends to balance overall prevalence rates on a sex specific basis.

- Heart failure admission rates appear to be steadily increasing in all industrialised countries, especially among older individuals. Overall, annual admission rates for 1990 ranged from 10-40 admissions/ 10000 population and increased to $>75$ admissions $/ 10000$ population in those aged $>65$ years.

- The cost of managing heart failure in the early 1990 s was estimated to be $1-2 \%$ of total health care expenditure. Because hospital care consumes a significant proportion of this expenditure, and rates of heart failure related hospitalisation have probably risen, this may be an underestimate of the current cost of heart failure.

- Heart failure is associated with an approximately $60 \%$ mortality rate within five years of diagnosis.

- The combination of increasing survival post acute myocardial infarction and increased longevity in western developed nations is likely to lead to an increase in the overall prevalence of heart failure.

ventricular ejection fraction: prevalence and mortality in a population-based cohort. J Am Coll Cardiol 1999;33:1948-55.

7. Cowie MR, Wood DA, Coats AJ, et al. Incidence and aetiology of heart failure; a population-based study. Eur Heart J 1999;20:421-8.

- A contemporary, London based, population survey of the incidence and new cases of chronic heart failure in men and women.

8. McMurray J, McDonagh T, Morrison CE, et al. Trends in hospitalization for heart failure in Scotland 1980-1990. Eur Heart J 1993:14:1158-62.

- A survey of heart failure related hospitalisations in Scotland during the period 1980 to 1990 . It represents the first European report of its kind.

9. Haldeman GA, Croft JB, Giles WH, et al. Hospitalization of patients with heart failure: national hospital discharge 
survey 1985-1995. Am Heart J 1999:137:352-60.

- A survey of heart failure related hospitalisations in the USA during the period 1985 to 1995 . It represents the most up to date report from an industrialised nation.

10. Kannel WB, Ho KK, Thom T. Changing epidemiological

features of cardiac failure. Eur Heart J 1994;72:S3-9.

- Using data from the long term surveillance of the Framingham cohorts, an important overview of the changing epidemiology and aetiology of heart failure.

11. The SOLVD Investigators. Effect of enalapril on survival in patients with reduced left ventricular ejection fractions and congestive heart failure. $N$ Engl J Med 1991;325:293-302.

12. Pitt B, Zannad F, Remme WJ, et al. The effect of spironolactone on morbidity and mortality in patients with severe heart failure. Randomized aldactone evaluation study investigators. N Engl J Med 1999;341:709-17.

13. The Digitalis Investigation Group. The effect of digoxin on mortality and morbidity in patients with heart failure. N Engl J Med 1997;336:525-33.

14. MERIT Investigators. Effect of metoprolol $C R / X L$ in chronic heart failure: metoprolol $\mathrm{CR} / \mathrm{XL}$ randomised intervention trial in congestive heart failure (Merit-HF). Lancet 1999;353:2001-7.

15. Packer M, Poole-Wilson PA, Armstrong PW, et al. Comparative effects of low and high doses of the angiotensin converting enzyme inhibitor, lisinopril, on morbidity and mortality in chronic heart failure. Circulation 1999;100:2312-18.
16. SOLVD Investigators. Natural history and patterns of current practice in heart failure. J Am Coll Cardiol 1993;4A:14A-19A.

17. Bart BA, Ertl G, Held P, et al. Contemporary management of patients with left ventricular systolic dysfunction. Results from the study of patients intolerant of converting enzyme inhibitors (SPICE) registry. Eur Heart $J$ 1999;20:1182-90.

- A clinical trial based registry that contains data from 105 study centres in eight countries in North America and Europe.

18. Ho KKL, Anderson KM, Karmel WB, et al. Survival after the onset of congestive heart failure in the Framingham heart study subjects. Circulation 1993:88:107-15.

- Another follow up report of the Framingham study describing extremely poor survival rates in those individuals diagnosed with chronic heart failure.

19. Rosamund WD, Chambless LE, Folsom AR, et al. Trends in the incidence of myocardial infarction and in mortality due to coronary artery disease. $N$ Engl J Med 1998;339:861-7.

20. Bonneux L, Barendregt JJ, Meeter K, et al. Estimating clinical morbidity due to ischaemic heart disease and congestive heart failure: the future rise of heart failure. $A m ~ J$ Public Health 1994;84:20-8.

- The first population based modelling exercise of its kind, this study estimated the future prevalence and associated burden of chronic heart failure in the Netherlands.

\section{website}

extra

Additional references appear on the Heart website

www.heartjnl.com 